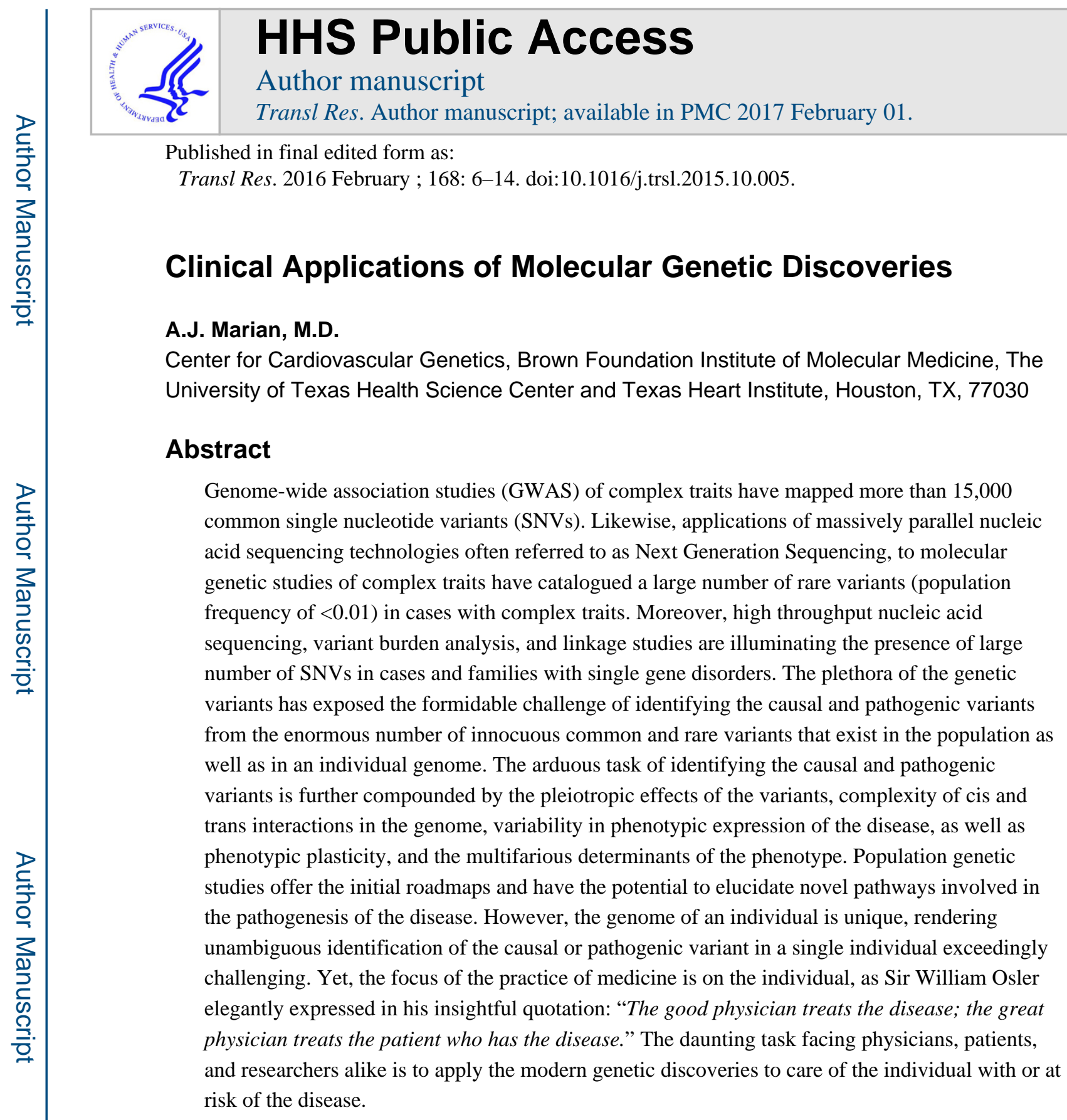

\title{
Keywords
}

Genetics; Single Nucleotide Variants; Genetic Testing; Mutation; Pathogenic Variants

Address for Correspondence: AJ Marian, M.D., Center for Cardiovascular Genetics, The Brown Foundation Institute of Molecular Medicine, The University of Texas Health Sciences Center, 6770 Bertner Street, Suite C900A, Houston, TX 77030, Phone: 713 500 2350, Fax: 713500 2320, Ali.J.Marian@uth.tmc.edu.

Publisher's Disclaimer: This is a PDF file of an unedited manuscript that has been accepted for publication. As a service to our customers we are providing this early version of the manuscript. The manuscript will undergo copyediting, typesetting, and review of the resulting proof before it is published in its final citable form. Please note that during the production process errors may be discovered which could affect the content, and all legal disclaimers that apply to the journal pertain.

Conflict of Interest: There is no conflict of interest to declare. The author has read the journal's policy on disclosure of potential conflicts of interest. 


\section{THE HUMAN GENOME}

The human nuclear genome (the genome) contains $~ 3.2$ billions base pairs, which are arranged in 22 pairs of somatic and 2 sex chromosomes. The genome is comprised of about 20,000 protein-coding genes containing $~ 180,000$ exons that collectively encompass about 35 million base pairs or about $1.1 \%$ of the genome. Pervasive transcription of the genome also produces more than 50,000 non-coding RNAs, including more than 2,000 microRNAs (1-3). MicroRNAs are involved in various biological processes, mostly as the "nudgers and tweaker" of genome management (4). As the name implies, the long non-coding RNAs (IncRNAs) do not encode proteins, although a few have been shown to code for small peptides (5-7). The functions of IncRNAs, with the exception of a few, have remained largely unknown (8). In addition, each gene transcribes multiple alternatively spliced mRNA species with one or two dominant splice variants $(9,10)$. Finally, the genome also contain over 100,000 regulatory elements that modulate gene expression and hence, the phenotype (11).

Genes and exons vary in size. Likewise, genes encompass a variable number of exons, which undergo considerable alternative splicing $(9,10)$. Dystrophin $(D M D)$, comprised of 2.2 million base pairs (Mbp), is the largest protein-coding gene in the genome. Titin (TTN) encompasses 364 exons and has the largest number of protein coding exons. It occupies more than 100,000 coding nucleotides and encodes a 34,350 amino acid protein. The nuclear genome is wrapped by histone protein complexes, which are modified by post-translational modifications, such as acetylation and methylation. These modifications regulate the chromatin state for accessibility of the transcriptional regulators for gene expression as well as genomic duplication during cellular proliferation (12). In addition to the nuclear genome, cell also contains mitochondrial genome (mtDNA), which is a circular molecule of $\sim 16,600$ base pairs long and 37 genes, which encode 13 proteins, 22 tRNAs, and 2 rRNAs. The vast majority of $\sim 3,000$ proteins involved in mitochondrial functions, however, are encoded by the nuclear genome.

The DNA polymerases and editing enzymes responsible for genomic duplication have an empirically determined error rate of $1.2 \times 10^{-8}$ per base pair (13-16). The rare error rate of DNA replication machinery leads to introduction of $\sim 30$ to 50 de novo variants during meiosis, i.e., such variants are absent in the parents. The de novo variants are predominantly of paternal origin and seem to be enriched in the functional and coding regions (15). Approximately $1.2 \%$ of the de novo variants reside in the exons, which is about $28.7 \%$ enrichment, as compared to the expected random distribution (15). Each meiosis also results in $\sim 3$ de novo small insertion/deletion (indel) and structural variations (SVs) (17).

At the population level, the rare error rate of DNA polymerases and editing enzymes translates to an enormous genetic diversity, as $\sim 30$ to 50 de novo variants during meiosis are introduced in each individual since evolution of the humans over 3.7 to 6.6 million years $(13,14)(15,16,18)$. The rapid expansion of the human population from approximately a few million people about 10,000 years ago to over 7 billion today has led to introduction of a vast number of DNA sequence variants (DSVs) into the population pool, which portrait the genetic diversity of the humans (19). Moreover, exponential growth of the human 
population during the last 400 generations has introduced an enormous number of new DSVs that have not yet spread in the population. Hence, such variants are either novel or rare. Given the rapid expansion of the human population during the last 10,000 years, the vast majority of the DSVs in the human population are rare, defined by a population frequency of $<0.01$. Thus, rare variants are more prevalent in the human population than the common variants. Empiric data show that approximately $86 \%$ of the variants identified, upon large-scale DNA sequencing, in defined populations have a minor allele frequency of $0.5 \%$ or less (20). As would be expected, rare variants are typically population-specific and their population frequency varies significantly across different ethnic backgrounds and even across different geographic regions $(20,21)$.

Because rare variants are typically of recent origin, they have been less subject to evolutionary selection pressure as compared to the ancient variants, which are typically common. Therefore, as compared to the common variants, rare variants are more likely to be biologically functional and exert larger effects on the phenotype. Large-scale DNA sequencing data show that approximately $96 \%$ of putatively functional variants in the population are rare variants (20). However, it is important to note that only a small fraction of the rare variants in each genome is functional and only a handful exerts clinically discernible effects. The vast majority of the rare variants do not impart a biologically significant or a clinically discernible effect. Nevertheless, high throughput DNA sequencing approaches have led to identification of a very large number of DSVs and enhanced the interest in identification and characterization of rare variants as genetic determinants of the clinical and biological phenotypes.

\section{UNIQUE NATURE OF EACH GENOME}

The rare error rate of DNA replication machinery introduces $\sim 30$ to 50 new variants in each genome rendering humans genetically very heterogeneous. Accordingly, the genome of each individual differs from the reference genome at about 3.5 million SNVs and several hundred thousands small indels and SVs, including large duplications, insertions, deletions, and rearrangements (22-25). Approximately 12,000 of the 3.5 million SNVs in each genome change the amino acids in the encoded proteins and are referred to as non-synonymous SNVs (nsSNVs) (22-25). Because non-synonymous SNVs alter protein structure, they have the potential to be functional, pathogenic, deleterious, and causal in diseases. In silico algorithms predict about $\sim 2$ to $10 \%$ of the nsSNVs in the human genome to be pathogenic, which means that several hundred to a thousand pathogenic SNVs are present in each genome (20,23). Exome and genome sequencing detects the presence of approximately 250 to 300 loss-of-function (LoF) SNVs in the annotated genes, including 25 to 35 stop codon variants, and 50 to 100 variants previously implicated in inherited disorders $(14,22,23)$. The genome also contains several hundred thousand small indels and SVs. SVs might involve more than a million nucleotides and affect copy number of the genes and loci (24, 26). Such variants are referred to as Copy Number Variants (CNVs). CNSs also exhibit significant diversity among different ethnic populations (27). Table 1 summarizes the approximate number of DSVs in each genome, as compared to the reference genome. 
Each genome comprises a large number of rare and novel variants. Approximately $20 \%$ of the SNVs identified in Dr. Venter's and Dr. Watson's genomes, which were sequenced in 2007 and 2008, respectively, were novel variants $(22,23)$. With increasingly availability of the whole exome sequencing (WES) and whole genome sequencing (WGS) data, the number of novel variants in each genome is expected to decrease but the number of rare variants is expected to remain much higher than the number of the common variants, adding complexity to interpretation of the genetic variants.

\section{GENETIC VARIANTS AND EXPRESSION OF THE PHENOTYPE}

The effect sizes of the DSVs in the genome are expected to follow a gradient that ranges from negligible to large $(28,29)$. On one end of the spectrum are the vast majority of the DSVs in the genome that are largely neutral and exert no discernible biological or clinical effects. On the opposite end are very rare variants that exert very large effect sizes and are responsible for single gene disorders with Mendelian patterns of inheritance. Over 3,600 genes responsible for single gene disorders with Mendelian patterns of inheritance have been identified. Likewise, over 15,000 SNVs for common complex diseases have been mapped through GWAS studies (www.genome.gov/).

Common variants, which are genotyped in the GWAS, generally exert modest effect sizes, which are typically clinically insignificant. For example, variants identified through the GWAS shift plasma levels of high-density lipoprotein-cholesterol (HDL-C) by 1 to $2 \mathrm{mg} / \mathrm{dl}$ and those of low-density lipoprotein-cholesterol (LDL-C) by $1-5 \mathrm{mg} / \mathrm{dl}$ (30-32). Similarly, common genetic variants associated with arterial blood pressure shift the systolic or diastolic blood pressure by $1 \mathrm{mmHg}$ or less $(33,34)$. Such differences are within the variability of quantification of the phenotype and in a given individual are clinically insignificant. There are a few notable exception, including two nsSNVs and a two-codon deletion variant in the APOL1 gene, which are common in African Americans but absent in the European population $(35,36)$. These common APOL1 variants are associated with chronic kidney disease, including non-diabetic nephropathy, focal segmental glomerulosclerosis, hypertension-attributed end-stage nephropathy, and progression to renal failure $(36,37)$. In contrast to the well-established role of APOL1 common variants in susceptibility to and progression of kidney disease, their role in cardiovascular disease remains unsettled $(38,39)$. Likewise, a missense variant (p.Glu6Val) in the $H B B$ gene encoding $\beta$-globin, which is common in Africans, is responsible for the common forms of sickle cell disease (40). The variant is also present in approximately $8 \%$ of African Americans, as carrier of sickle cell trait (40). Another notable example of a common variant with a large biological effect size is the insertion/deletion polymorphism in the $A C E$ gene, which accounts for about half of the variance in the plasma levels of ACE in the population (41). However, despite its large biological effects on the intermediate phenotype, clinical significance of the insertion/ deletion polymorphism in the $A C E$ gene is less certain.

The weak effect sizes of the common variants render them almost unusable in predicting the disease risk. Often a genetic risk score, derived from multiple common SNVs, are generated to calculate the disease risk. Such scores are based on a number of assumptions that require biological evidence, which are often not met. Consequently, clinical utility of genetic risk 
scores is also limited, albeit is often being used as a research tool but seldom clinically. It is important to note, however, that the weak effect of the common DSVs on the phenotype do not negate the significance of the genetic discoveries in providing insights into the molecular basis of the diseases and deciphering novel pathways. This is exemplified by the effects of common variants in the HMGCR gene, encoding 3-hydroxy-3-methylglutaryl-coenzyme A reductase, a target of statins. The variants shift plasma LDL-C levels by $2-3 \mathrm{mg} / \mathrm{dl}$, and yet, statins by inhibiting HMGCR protein effectively reduce plasma LDL-C levels and adverse cardiovascular outcomes.

The vast majority of the rare variants also do not exert discernible biological or clinical effects. However, a rare variant is more likely to be pathogenic than a common variant (30). Approximately $96 \%$ of the biologically functional variants, as predicted by bioinformatics algorithms, are rare variants (20). Likewise, each genome contains several thousands rare variants that are expected to be functional. However, only a fraction of the functional variants are involved in the pathogenesis of the phenotype or are deleterious, defined as variants that reduce reproductive fitness (42). It is also important to note that a notable fraction of the previously considered causal variants are rare, uncommon and even common variants in the general population and no longer could be considered causal variants. This misclassification was discovered with the increasing availability of the large-scale DNA sequencing datasets detecting such variants in the general population at a frequency higher than anticipated for a disease- causing variant (43-45). A recent low coverage WGS of 179 individuals detected 40-110 variants in each genome that were classified as disease-causing mutations in the Human Gene Mutation Database (HGMD) database, which reflects an overestimation of the disease-causing variants (43). Correcting for such mis-classification, it is estimated that each genome carries $\sim 400$ damaging variants and $\sim 2$ bona fide disease variants (43).

\section{CLINICAL HIERARCHY OF THE GENETIC VARIANTS}

The abundance of the variants in each genome poses a major challenge to physician and investigators alike in accurate identification of the causal and pathogenic variants (42). Distinct categorization of the clinically significant variants is exceedingly challenging and subject to false positive and false negative calls. Bioinformatics algorithms are useful but alone unreliable in predicts pathogenicity of the variants and the platforms often differ in their prediction of pathogenicity. In addition, there is considerable variability in the phenotypic expression of the variants as well as plasticity of the phenotype that further compound an accurate ascertainment of pathogenicity of the variants. The personal genome data provide insightful indication of complexity of predicting the pathogenic or deleterious variants simply based on bioinformatics algorithms or their previous implications in the disease. As an example, Dr. James Watson's genome comprises about 3.3 million SNVs, including more than $10,000 \mathrm{nsSNVs}$, of which $7.3 \%$ are predicted to be "probably damaging" and 310 nsSNVs are in genes previously implicated in human diseases (23). Likewise, Dr. Watson's genome contains 32 variants that have already been linked to human diseases in the HGMD, and 10 variants that are linked to highly penetrant Mendelian recessive diseases (23). Yet, Dr. Watson, as known, is free from such medical conditions. The data not only point to phenotypic variability of the genetic variants but also suggest an 
overcall in the published data in categorization of the pathogenic and disease-causing variants.

A phenotype results from the confluence of a large number of genetic and environmental factors, each contributing modestly to its expression (46). Genetic variants are important determinants of the phenotype but their effect sizes vary from being negligible to large including those exhibiting a near complete penetrance. At present the focus is mostly on coding variants that alter protein structure. The role of variants that regulate gene expression is not nearly as well explored. Based on the prevalence of the variants and their effect sizes, DSVs might simply categorized into two broad categories of functional variants comprised of causal, pathogenic, and functional only variants; and variants with unknown significance, as follows (Figure 1):

\section{Functional variants}

Functional variants are those that have been characterized and shown to affect expression and/or functions of the transcripts and the encoded proteins. Functional variants might be categorized into three groups of causal, pathogenic, and functional only variants.

Causal variants-The conventional definition of causality - as originally defined by Robert Koch in the $19^{\text {th }}$ century (discussed in $(47,48)$ ), seldom pertains to the genetic variants. In its strict definition, however, the causal variant is necessary and sufficient to cause the phenotype, albeit with a variable phenotypic expression, as the phenotype is also influenced by the modifying genetic and non-genetic factors. Thus, in a sense, causality does not apply to complex phenotypes, as a variant is neither necessary nor sufficient to cause the complex phenotype. However, in single gene disorders with Mendelian patterns of inheritance, variants that exhibit high penetrance and co-segregate with inheritance of the phenotype in large families might fulfill the classic definition of causality. Likewise, de novo mutations that perfectly co-segregate with inheritance of the phenotype in members of large families also benefit from firm evidence of causality. Identification of the causal variant in such scenarios enables cascade genetic screening of all family members and hence, categorical distinction of those who have inherited the variant and hence, are at the risk of the disease from those who have not and hence, are at the same risk as the general population. This, however, often is not the case, as the families are typically small in size and the penetrance is incomplete. Large-scale exome sequencing studies in the general population show the presence of rare including putatively functional variants in almost all genes, including genes known to cause Mendelian disorders (http://exac.broadinstitute.org/). Often these rare and uncommon variants are more common than the prevalence of the single gene disorder or the expected prevalence of the disease-causing variants (43-45). Thus, in most situations, unambiguous assignment of causality in small families and individual cases with the phenotype of a single gene disorder is exceedingly challenging.

Establishing causality is even more challenging for complex traits because of the multiplicity of the etiological determinants and the small effect size of each. To infer causality in complex traits, DSVs are often used as instrumental variable in the statistical analysis (49). This is based on the principle that DSVs are assorted randomly during meiosis 
and hence, are not subject to confounding effects of the environmental factors, which are to be distributed randomly among the genotypes(49). The approach, which is referred to as Mendelian randomization or instrumental variable analysis with genetic instruments, is best applicable for DSVs with large effect sizes, which is seldom the case for the complex traits. In general, DSVs, because of their modest effect sizes, are weak instrumental variables, increasing the chance of a null finding in Mendelian randomization studies and making causal inference difficult. In vitro and in vivo studies in model organisms could establish functionality of the variants and are complementary to the clinical genetic data but are insufficient to establish causality unambiguously $(48,50)$.

Availability of large databases of genetic variants such as ExAC (http:// exac.broadinstitute.org/) offers the opportunity to assess population prevalence of the variants. Accordingly, a number of nsSNVs that were previously considered causal are found to be present in the general population at a frequency higher the expected for a disease-causing variant (43-45). It also merit noting that identification of a nsSNV in a gene previously identified as a causal gene for a single gene disorder alone is insufficient to indicate causality. As indicated earlier, unambiguous identification of the causal variant in a single case or small families is exceedingly difficult, because of the presence of such variants in the general population and in each genome. In vitro and in vivo data could offer supportive data and establish functionality of the variants under the experimental conditions but not necessarily the causality (48). Nevertheless, a reasonable assumption of causality in small families and cases could be made if the analogous elements of Koch's postulates are fulfilled as follows:

- Statistical evidence: Evidence of co-segregation of the variants with the phenotype in members of the family, while considering incomplete penetrance and the small size of the family, which hinder from robust linkage analysis. Similarly, robust statistical evidence for enrichment of the rare variants in the cases with the phenotype is necessary.

- Biological evidence: Evidence for functionality of the variant in in vitro and in vivo studies. Likewise, introduction of the variant into a model organism must induce a phenotype that somewhat resembles the phenotype in humans;

- Phenotypic rescue: Elimination of the causal variant or silencing of its expression in the model organism has to lead to reversal and resolution of the phenotype.

Pathogenic variants-A subset of biologically functional variants impart effect sizes that are large enough to influence pathogenesis of the phenotype and hence, they are considered pathogenic variants. They are neither sufficient nor necessary to cause the phenotype but in aggregate might cause the phenotype or individually impact expression of the phenotype. Pathogenic variants are typically enriched in the cases with the phenotype as opposed to controls. Therefore, they might be also found in the general population and individuals without the phenotype. Pathogenic variants are typically rare variants that lead to premature truncation, gain- or loss-of-stop codon, alter RNA splicing, and affect protein structure and function. Evidence for their pathogenic role, in addition to statistical evidence of enrichment in the cases, is further supported by functional studies. Variants in the coding regions are the 
primary focus as the pathogenic variants and those in the non-coding regions are largely uncharacterized. Only a small fraction of the variants identified in GWAS are pathogenic variants and the vast majority is likely in linkage disequilibrium or interacts with the true pathogenic variants.

Functional only variants-The category includes variants that have been shown to be functional but are not associated with a phenotype or exert small effect sizes that are functionally and clinically not discernible. This category includes the majority of the functional variants in the genome. Evidently with the increasing availability of the genotypephenotype data, functional variants might be re-classified as pathogenic variants.

\section{Variants of unknown significance}

The vast majority of the DSVs in the exome or genome are variants that have not been associated with biological functions or clinical phenotypes. This category comprises a large number of variants that are identified through GWAS, which are not known to have biological functions and might be in linkage disequilibrium with the pathogenic variants. Unfortunately, identification of the pathogenic variants for complex traits, based on the results of GWAS, has been exceedingly challenging. This is in part because of the complexity of the genome and the presence of a large number of cis and trans interactions among chromosomal regions (51-53). Consequently, clinical utility of the variants identified through GWAS has been limited. Nevertheless, the promise of the approach is in elucidation of the underlying responsible mechanism(s), which has the potential to lead to discovery new pathways and hence, new preventive and therapeutic targets. This point is illustrated by the discovery of SORT1 locus in GWAS of LDL-C and myocardial infarction $(54,55)$. SORT1 encodes Sortilin 1, which regulates lipoprotein metabolism (56). It is currently being investigated as a potential drug target for treatment of hypercholesterolemia.

\section{CLINICAL IMPLICATIONS OF THE GENETIC DISCOVERIES}

There is a considerable interest in training the clinical practitioners in utilizing genetic testing and applying the genetic discoveries to the practice of medicine (57). Physicians are expected to muster an adequate level of knowledge in actionable genetic findings (57). They are also expected to recognize familial inheritance, aggregation of the phenotype in families, co-segregation of the genetic variants with inheritance of the phenotype, causal and pathogenic variants, and be able to apply the knowledge to genetic screening of those at risk. These initial re-tooling of the physicians is expected to enable building a partial bridge to cover the gap between the genetic discoveries and medical practice. Application of the genetics and genomics to the practice of medicine, nevertheless, is expected to be quite complex, for reasons discussed above, requiring a level of sophistication in knowledge that might not be easily mustered.

A clinical phenotype is the consequence of multiplex, intertwined and stochastic interactions among various determinants, including genetic and environmental factors. Consequently, ascertaining a phenotypic outcome based on a single determinant, whether genetic or otherwise, is unrealistic with the exception of rare circumstances. It is particularly challenging for the complex traits, whereby each determinant contributes modestly to the 
phenotype. It also merit noting that the effects of the genetic variants diminish with the remoteness of the phenotype to genotype because of the diluting effects of a large number of competing factors. Even in single gene-disorders, whereby the effect size of the causal variant is relatively large, accurate prediction of the clinical outcomes or severity of the phenotype is constrained by numerous genetic and environmental factors.

Genetic studies are most powerful in the familial setting. Thus, as much as possible, genetic and phenotypic data must be gathered in all family members to increase garnering meaningful information and gaining insight into the pathogenic role of the variants. In familial settings, cascade genetic testing could offer pre-clinical identifications of those at risk. Such preclinical diagnosis could lead to interventions to reduce and eliminate the risk of the disease, based on insights gained in understanding the underlying mechanisms of the disease. Likewise, in a subset of patients, genetic-based diagnosis could lead to accurate diagnosis, and elimination of the phenocopy conditions, therefore, accurate identification and proper treatment. Nevertheless, primacy of genetic discoveries in clinical medicine is not in genetic-based diagnosis, risk stratification, or prognostication, but it is rather in providing insights into the fundamental mechanisms that govern the pathogenesis of the disease. It is this fundamental understanding that could ultimately lead to prevention and cure of human diseases. A notable example is the discovery of PCSK9, which was mapped in 2003 as a cause of autosomal dominant hypercholesterolemia (58). This totally new discovery, within a decade led to development and clinical applications of PCSK9 inhibitors as exceedingly effective therapies for lowering LDL-C and reducing adverse cardiovascular outcomes $(59,60)$. Thus, genetic discoveries provide the best means to delineate the fundamental basis of the human disease, enabling specific targeting of the responsible pathways for preventive and therapeutic gains. Table 2 lists some of the potential clinical applications of the molecular genetic discoveries.

\section{CONCLUSIONS}

Recent technological advances have afforded the opportunity to decipher the genetic architecture of the genome, which has revealed the plethora of DSVs, including a large number of putatively functional and pathogenic rare and uncommon variants. The daunting task facing physicians and the investigators is to identify those variants that are causal or at least contribute to the pathogenesis of the phenotype (pathogenic variants). The current training and expertise of the clinicians or geneticists is inadequate for garnering unequivocal medical information from the genetic data, if at all possible. In-depth knowledge in genetics as well as clinical medicine is required in complementation with ongoing laboratory and clinical investigation to define the clinical significance of the DSVs in the genome, while realizing that genetic factors are only part of the multitude of determinants of the clinical phenotypes. The approach could enable the physician to conform with Sir William Osler's adage that "the great physician treats the patient who has the disease".

\section{Acknowledgment}

None

FUNDING SOURCES: 
AJ Marian was supported in part by grants from NIH, National Heart, Lung and Blood Institute (NHLBI, R01 HL088498 and R34 HL105563), Leducq Foundation (14 CVD 03), Roderick MacDonald Foundation (13RDM005), TexGen Fund from Greater Houston Community Foundation and George and Mary Josephine Hamman Foundation.

\section{REFERENCES}

1. Iyer MK, Niknafs YS, Malik R, Singhal U, Sahu A, Hosono Y, et al. The landscape of long noncoding RNAs in the human transcriptome. Nature genetics. 2015; 47(3):199-208. [PubMed: 25599403]

2. Londin E, Loher P, Telonis AG, Quann K, Clark P, Jing Y, et al. Analysis of 13 cell types reveals evidence for the expression of numerous novel primate- and tissue-specific microRNAs. Proceedings of the National Academy of Sciences of the United States of America. 2015; 112(10):E1106-E1115. [PubMed: 25713380]

3. Hangauer MJ, Vaughn IW, McManus MT. Pervasive transcription of the human genome produces thousands of previously unidentified long intergenic noncoding RNAs. PLoS Genet. 2013; 9(6):e1003569. [PubMed: 23818866]

4. Bird A. Genome biology: not drowning but waving. Cell. 2013; 154(5):951-952. [PubMed: 23993086]

5. Magny EG, Pueyo JI, Pearl FM, Cespedes MA, Niven JE, Bishop SA, et al. Conserved regulation of cardiac calcium uptake by peptides encoded in small open reading frames. Science. 2013; 341(6150):1116-1120. [PubMed: 23970561]

6. Nelson BR, Anderson DM, Olson EN. Small open reading frames pack a big punch in cardiac calcium regulation. Circulation research. 2014; 114(1):18-20. [PubMed: 24385504]

7. Anderson DM, Anderson KM, Chang CL, Makarewich CA, Nelson BR, McAnally JR, et al. A micropeptide encoded by a putative long noncoding RNA regulates muscle performance. Cell. 2015; 160(4):595-606. [PubMed: 25640239]

8. Rizki G, Boyer LA. Lncing epigenetic control of transcription to cardiovascular development and disease. Circulation research. 2015; 117(2):192-206. [PubMed: 26139858]

9. Djebali S, Davis CA, Merkel A, Dobin A, Lassmann T, Mortazavi A, et al. Landscape of transcription in human cells. Nature. 2012; 489(7414):101-108. [PubMed: 22955620]

10. Mele M, Ferreira PG, Reverter F, DeLuca DS, Monlong J, Sammeth M, et al. Human genomics. The human transcriptome across tissues and individuals. Science. 2015; 348(6235):660-665. [PubMed: 25954002]

11. Consortium EP. An integrated encyclopedia of DNA elements in the human genome. Nature. 2012; 489(7414):57-74. [PubMed: 22955616]

12. Rivera CM, Ren B. Mapping human epigenomes. Cell. 2013; 155(1):39-55. [PubMed: 24074860]

13. Kong A, Frigge ML, Masson G, Besenbacher S, Sulem P, Magnusson G, et al. Rate of de novo mutations and the importance of father's age to disease risk. Nature. 2012; 488(7412):471-475. [PubMed: 22914163]

14. Abecasis GR, Altshuler D, Auton A, Brooks LD, Durbin RM, et al. Genomes Project C. A map of human genome variation from population-scale sequencing. Nature. 2010; 467(7319):1061-1073. [PubMed: 20981092]

15. Francioli LC, Polak PP, Koren A, Menelaou A, Chun S, Renkens I, et al. Genome-wide patterns and properties of de novo mutations in humans. Nature genetics. 2015; 47(7):822-826. [PubMed: 25985141]

16. Conrad DF, Keebler JE, DePristo MA, Lindsay SJ, Zhang Y, Casals F, et al. Variation in genomewide mutation rates within and between human families. Nature genetics. 2011; 43(7):712-714. [PubMed: 21666693]

17. Kloosterman WP, Francioli LC, Hormozdiari F, Marschall T, Hehir-Kwa JY, Abdellaoui A, et al. Characteristics of de novo structural changes in the human genome. Genome Res. 2015; 25(6): 792-801. [PubMed: 25883321] 
18. Sun JX, Helgason A, Masson G, Ebenesersdottir SS, Li H, Mallick S, et al. A direct characterization of human mutation based on microsatellites. Nature genetics. 2012; 44(10):11611165. [PubMed: 22922873]

19. Keinan A, Clark AG. Recent explosive human population growth has resulted in an excess of rare genetic variants. Science. 2012; 336(6082):740-743. [PubMed: 22582263]

20. Tennessen JA, Bigham AW, O'Connor TD, Fu W, Kenny EE, Gravel S, et al. Evolution and functional impact of rare coding variation from deep sequencing of human exomes. Science. 2012; 337(6090):64-69. [PubMed: 22604720]

21. Novembre J, Johnson T, Bryc K, Kutalik Z, Boyko AR, Auton A, et al. Genes mirror geography within Europe. Nature. 2008; 456(7218):98-101. [PubMed: 18758442]

22. Levy S, Sutton G, Ng PC, Feuk L, Halpern AL, Walenz BP, et al. The diploid genome sequence of an individual human. PLoS Biol. 2007; 5(10):e254. [PubMed: 17803354]

23. Wheeler DA, Srinivasan M, Egholm M, Shen Y, Chen L, McGuire A, et al. The complete genome of an individual by massively parallel DNA sequencing. Nature. 2008; 452(7189):872-876. [PubMed: 18421352]

24. Kidd JM, Cooper GM, Donahue WF, Hayden HS, Sampas N, Graves T, et al. Mapping and sequencing of structural variation from eight human genomes. Nature. 2008; 453(7191):56-64. [PubMed: 18451855]

25. Wang J, Wang W, Li R, Li Y, Tian G, Goodman L, et al. The diploid genome sequence of an Asian individual. Nature. 2008; 456(7218):60-65. [PubMed: 18987735]

26. Korbel JO, Urban AE, Affourtit JP, Godwin B, Grubert F, Simons JF, et al. Paired-end mapping reveals extensive structural variation in the human genome. Science. 2007; 318(5849):420-426. [PubMed: 17901297]

27. Sudmant PH, Mallick S, Nelson BJ, Hormozdiari F, Krumm N, Huddleston J, et al. Global diversity, population stratification, and selection of human copy-number variation. Science. 2015; 349(6253):aab3761. [PubMed: 26249230]

28. Marian AJ. Nature's genetic gradients and the clinical phenotype. Circulation Cardiovascular genetics. 2009; 2(6):537-539. [PubMed: 20031631]

29. Marian AJ. Molecular genetic studies of complex phenotypes. Translational research : the journal of laboratory and clinical medicine. 2012; 159(2):64-79. [PubMed: 22243791]

30. Lange LA, Hu Y, Zhang H, Xue C, Schmidt EM, Tang ZZ, et al. Whole-exome sequencing identifies rare and low-frequency coding variants associated with LDL cholesterol. American journal of human genetics. 2014; 94(2):233-245. [PubMed: 24507775]

31. Kathiresan S, Willer CJ, Peloso GM, Demissie S, Musunuru K, Schadt EE, et al. Common variants at 30 loci contribute to polygenic dyslipidemia. Nature genetics. 2009; 41(1):56-65. [PubMed: 19060906]

32. Willer CJ, Schmidt EM, Sengupta S, Peloso GM, Gustafsson S, et al. Global Lipids Genetics C. Discovery and refinement of loci associated with lipid levels. Nature genetics. 2013; 45(11):12741283. [PubMed: 24097068]

33. Wain LV, Verwoert GC, O'Reilly PF, Shi G, Johnson T, Johnson AD, et al. Genome-wide association study identifies six new loci influencing pulse pressure and mean arterial pressure. Nature genetics. 2011; 43(10):1005-1011. [PubMed: 21909110]

34. Ehret GB, Munroe PB, Rice KM, Bochud M, Johnson AD, et al. International Consortium for Blood Pressure Genome-Wide Association S. Genetic variants in novel pathways influence blood pressure and cardiovascular disease risk. Nature. 2011; 478(7367):103-109. [PubMed: 21909115]

35. Tzur S, Rosset S, Shemer R, Yudkovsky G, Selig S, Tarekegn A, et al. Missense mutations in the APOL1 gene are highly associated with end stage kidney disease risk previously attributed to the MYH9 gene. Hum Genet. 2010; 128(3):345-350. [PubMed: 20635188]

36. Genovese G, Friedman DJ, Ross MD, Lecordier L, Uzureau P, Freedman BI, et al. Association of trypanolytic ApoL1 variants with kidney disease in African Americans. Science. 2010; 329(5993): 841-845. [PubMed: 20647424]

37. Parsa A, Kao WH, Xie D, Astor BC, Li M, Hsu CY, et al. APOL1 risk variants, race, and progression of chronic kidney disease. The New England journal of medicine. 2013; 369(23): 2183-2196. [PubMed: 24206458] 
38. Ito K, Bick AG, Flannick J, Friedman DJ, Genovese G, Parfenov MG, et al. Increased burden of cardiovascular disease in carriers of APOL1 genetic variants. Circulation research. 2014; 114(5): 845-850. [PubMed: 24379297]

39. Langefeld CD, Divers J, Pajewski NM, Hawfield AT, Reboussin DM, Bild DE, et al. Apolipoprotein L1 gene variants associate with prevalent kidney but not prevalent cardiovascular disease in the Systolic Blood Pressure Intervention Trial. Kidney Int. 2015; 87(1):169-175. [PubMed: 25029429]

40. Forget BG, Bunn HF. Classification of the disorders of hemoglobin. Cold Spring Harb Perspect Med. 2013; 3(2):a011684. [PubMed: 23378597]

41. Villard E, Tiret L, Visvikis S, Rakotovao R, Cambien F, Soubrier F. Identification of new polymorphisms of the angiotensin I-converting enzyme (ACE) gene, and study of their relationship to plasma ACE levels by two-QTL segregation-linkage analysis. American journal of human genetics. 1996; 58(6):1268-1278. [PubMed: 8651305]

42. MacArthur DG, Manolio TA, Dimmock DP, Rehm HL, Shendure J, Abecasis GR, et al. Guidelines for investigating causality of sequence variants in human disease. Nature. 2014; 508(7497):469_ 476. [PubMed: 24759409]

43. Xue Y, Chen Y, Ayub Q, Huang N, Ball EV, Mort M, et al. Deleterious- and disease-allele prevalence in healthy individuals: insights from current predictions, mutation databases, and population-scale resequencing. American journal of human genetics. 2012; 91(6):1022-1032. [PubMed: 23217326]

44. Bell CJ, Dinwiddie DL, Miller NA, Hateley SL, Ganusova EE, Mudge J, et al. Carrier testing for severe childhood recessive diseases by next-generation sequencing. Science translational medicine. 2011; 3(65):65ra4.

45. Norton N, Robertson PD, Rieder MJ, Zuchner S, Rampersaud E, Martin E, et al. Evaluating pathogenicity of rare variants from dilated cardiomyopathy in the exome era. Circulation Cardiovascular genetics. 2012; 5(2):167-174. [PubMed: 22337857]

46. Marian AJ, Belmont J. Strategic approaches to unraveling genetic causes of cardiovascular diseases. Circulation research. 2011; 108(10):1252-1269. [PubMed: 21566222]

47. Marian AJ, Roberts R. On Koch's postulates, causality and genetics of cardiomyopathies. Journal of molecular and cellular cardiology. 2002; 34(8):971-974. [PubMed: 12234766]

48. Marian AJ. Causality in genetics: the gradient of genetic effects and back to Koch's postulates of causality. Circulation research. 2014; 114(2):e18-e21. [PubMed: 24436434]

49. Wehby GL, Ohsfeldt RL, Murray JC. 'Mendelian randomization' equals instrumental variable analysis with genetic instruments. Stat Med. 2008; 27(15):2745-2749. [PubMed: 18344186]

50. Chen SN, Czernuszewicz G, Tan Y, Lombardi R, Jin J, Willerson JT, et al. Human molecular genetic and functional studies identify TRIM63, encoding Muscle RING Finger Protein 1, as a novel gene for human hypertrophic cardiomyopathy. Circulation research. 2012; 111(7):907-919. [PubMed: 22821932]

51. Denker A, de Laat W. A Long-Distance Chromatin Affair. Cell. 2015; 162(5):942-943. [PubMed: 26317462]

52. Waszak SM, Delaneau O, Gschwind AR, Kilpinen H, Raghav SK, Witwicki RM, et al. Population Variation and Genetic Control of Modular Chromatin Architecture in Humans. Cell. 2015; 162(5): 1039-1050. [PubMed: 26300124]

53. Grubert F, Zaugg JB, Kasowski M, Ursu O, Spacek DV, Martin AR, et al. Genetic Control of Chromatin States in Humans Involves Local and Distal Chromosomal Interactions. Cell. 2015; 162(5):1051-1065. [PubMed: 26300125]

54. Kathiresan S, Melander O, Guiducci C, Surti A, Burtt NP, Rieder MJ, et al. Six new loci associated with blood low-density lipoprotein cholesterol, high-density lipoprotein cholesterol or triglycerides in humans. Nature genetics. 2008; 40(2):189-197. [PubMed: 18193044]

55. Kathiresan S, Voight BF, Purcell S, Musunuru K, Ardissino D, et al. Myocardial Infarction Genetics C. Genome-wide association of early-onset myocardial infarction with single nucleotide polymorphisms and copy number variants. Nature genetics. 2009; 41(3):334-341. [PubMed: 19198609] 
56. Strong A, Patel K, Rader DJ. Sortilin and lipoprotein metabolism: making sense out of complexity. Curr Opin Lipidol. 2014; 25(5):350-357. [PubMed: 25101658]

57. Korf BR, Berry AB, Limson M, Marian AJ, Murray MF, O'Rourke PP, et al. Framework for development of physician competencies in genomic medicine: report of the Competencies Working Group of the Inter-Society Coordinating Committee for Physician Education in Genomics. Genetics in medicine : official journal of the American College of Medical Genetics. 2014

58. Abifadel M, Varret M, Rabes JP, Allard D, Ouguerram K, Devillers M, et al. Mutations in PCSK9 cause autosomal dominant hypercholesterolemia. Nature genetics. 2003; 34(2):154-156. [PubMed: 12730697]

59. Sabatine MS, Giugliano RP, Wiviott SD, Raal FJ, Blom DJ, Robinson J, et al. Efficacy and safety of evolocumab in reducing lipids and cardiovascular events. The New England journal of medicine. 2015; 372(16):1500-1509. [PubMed: 25773607]

60. Robinson JG, Farnier M, Krempf M, Bergeron J, Luc G, Averna M, et al. Efficacy and safety of alirocumab in reducing lipids and cardiovascular events. The New England journal of medicine. 2015; 372(16):1489-1499. [PubMed: 25773378] 


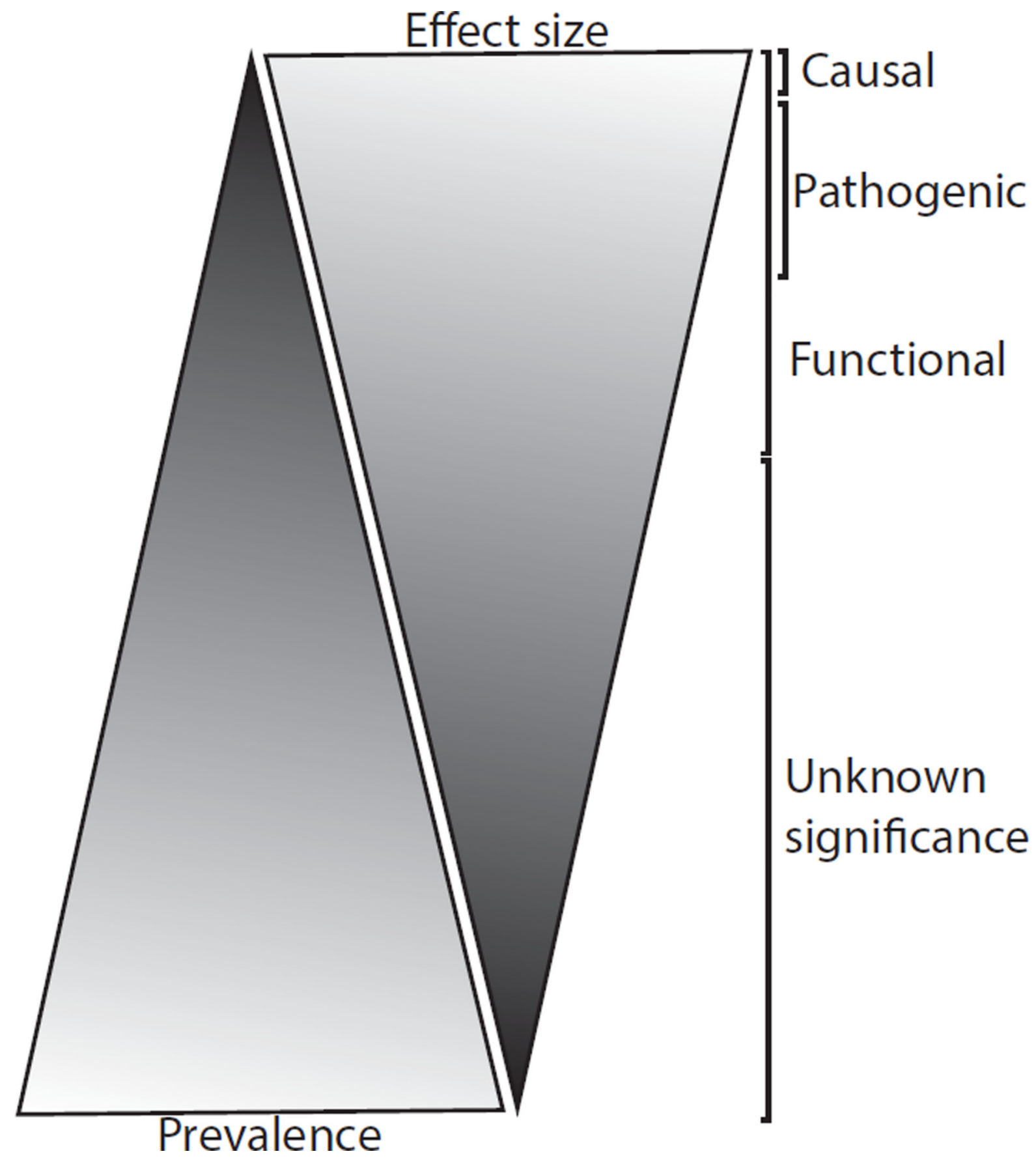

Figure 1. Gradients of prevalence of the variants, their effect sizes and clinical impacts

Prevalence of each category of DNA sequence variants (DSVs) in the genome along with their effect sizes are shown. Causal variants are rare in each genome and exert the largest effect sizes. Pathogenic variants are very uncommon, impart discernible effect sizes, and contribute to the pathogenesis of the phenotype. A large number of functional variants in the genome are not linked to a phenotype, while the vast majority of the DSVs in the genome 
are not known to exert biological functions or biological effects and hence, are variants with unknown functions. 


\section{TABLE 1}

Prevalence and Type of DNA Sequence Variants in the Human Genome

\begin{tabular}{ll} 
Number of base pairs & $3.2 \times 10^{9}$ \\
Size of exome (base pairs) & $35 \times 10^{6}$ \\
Number of protein-coding genes & 20,000 \\
Number of DNA Sequence Variants (DSVs) & $4 \times 10^{6}$ \\
Number of Single Nucleotide Variants (SNVs) & $3.5 \times 10^{6}$ \\
Number of Non-synonymous SNVs (nsSNVs) & 12,000 \\
Number of Loss-of-Function (LoF) heterozygous variants & $100-120$ \\
Variants associated with inherited diseases & $50-100$ \\
De novo variants (absent in the parents) & $30-50$ \\
\hline
\end{tabular}

Transl Res. Author manuscript; available in PMC 2017 February 01. 


\section{TABLE 2}

\section{Clinical Applications of Molecular Genetic Discoveries}

\begin{tabular}{|c|c|}
\hline Genetic testing & $\begin{array}{l}\text { - } \quad \text { Best applied in familial setting and through trio or cascade screening } \\
\text { - } \quad \text { Best applied in single gene disorders with Mendelian patterns of inheritance } \\
\text { - } \quad \text { Preclinical diagnosis in familial setting enabling interventions to reduce the risk } \\
\text { - } \quad \text { Unambiguous assignment of causality is almost impossible in a single individual in most situations } \\
\text { - } \quad \text { Limited applications in complex phenotypes, whether as a single variant or as a genetic risk score }\end{array}$ \\
\hline $\begin{array}{l}\text { Accurate diagnosis and } \\
\text { disease classification }\end{array}$ & $\begin{array}{l}\text { - Distinction of the disease from phenocopy conditions, which enables implementation of proper } \\
\text { treatment } \\
\text { Possible genetic-based categorization of the disease, which might enable rendering appropriate } \\
\text { therapy }\end{array}$ \\
\hline Prognostication & - With few exceptions, limited impact beyond the clinical means and often unreliable \\
\hline Response to therapy & $\begin{array}{l}\text { - } \quad \text { Early applications in cancer therapy } \\
\text { Potential for individualized therapy and prevention of side effects, albeit not proven to be superior } \\
\text { to conventional medical approaches }\end{array}$ \\
\hline $\begin{array}{l}\text { Insight into fundamental } \\
\text { mechanism(s) of disease }\end{array}$ & $\begin{array}{l}\text { This is likely the primacy of the genetic data, enabling discovery of new mechanisms and targets } \\
\text { for prevention and treatment }\end{array}$ \\
\hline
\end{tabular}

Transl Res. Author manuscript; available in PMC 2017 February 01. 